\title{
On convergence of tracking differentiator
}

\author{
Bao-Zhu Guo ${ }^{\text {abcd }}$ and Zhi-Liang Zhao ${ }^{\text {d* }}$ \\ ${ }^{a}$ Academy of Mathematics and Systems Science, Academia Sinica, Beijing 100190, P.R. China; \\ ${ }^{b}$ School of Computational and Applied Mathematics, University of the Witwatersrand, Wits 2050, Johannesburg, \\ South Africa; ${ }^{c}$ School of Mathematical Sciences, Shanxi University, Taiyuan 030006, P.R. China; \\ ${ }^{d}$ Department of Mathematics, University of Science and Technology of China, Hefei 230026, Anhui, P.R. China
}

(Received 2 November 2010; final version received 6 March 2011)

\begin{abstract}
The tracking differentiator was first proposed by Han in 1989 and the proof of convergence was presented the first time in Han and Wang (Han, J.Q., and Wang, W. (1994), 'Nonlinear Tracking-differentiator', Journal of Systems Science and Mathematical Science, 14, 177-183 (in Chinese)). Unfortunately, the proof there is incomplete. This problem has been open for over two decades. In this article, we give a rigorous proof under some additional conditions. An application for online estimation of the unknown frequencies for the finite sum of the sinusoidal signals is presented. The numerical simulations illustrate the effectiveness of the estimation for both linear and nonlinear tracking differentiators.
\end{abstract}

Keywords: tracking differentiator; stability

AMS Subject Classifications: 34D20; 34K35; 93C15; 94A12

\section{Introduction}

It is known that the powerful yet primitive proportional-integral-derivative (PID) control law developed in the period of the 1920s-1940s in the last century is still playing very important role in modern engineering control practice (Silva, Datta, and Bhattacharyya 2002; Han 2009). However, because of the noise sensitivity, the derivative control is not always physically implementable for most of control systems. A noise-tolerant tracking differentiator was proposed over two decades ago by Han (1989). Subsequently, many engineering applications have been made (see e.g. Emaru and Tsuchiya 2003; Sun and Gao 2005; Su, Zheng, Dong, and Duan 2005; Su et al. 2005). However, except the linear case that is proved in Guo, Han, and Xi (2002), a rigorous mathematical proof is still lacking for nonlinear tracking differentiators. Although the first effort was made almost 20 years ago in Han and Wang (1994), the proof there is only true for constant signal (see also Emaru and Tsuchiya (2003)), and the proof for general signal through the approximation of step functions is not valid as we shall indicate below.

Since the proof of Theorem 1 of Han and Wang (1994) is reproduced in the literature ( $\mathrm{Su}$ et al. 2005) as its Theorem 1, we refer it as Theorem 1.1 below.
Theorem 1.1 (Su et al. 2005): If any solution of the system following

$$
\left\{\begin{array}{l}
\dot{z}_{1}(t)=z_{2}(t) \\
\dot{z}_{2}(t)=f\left(z_{1}(t), z_{2}(t)\right)
\end{array}\right.
$$

satisfies $\lim _{t \rightarrow \infty}\left(z_{1}(t) z_{2}(t)\right)=0$, then for any bounded integral function $v(t)$ and any constant $T>0$, the solution of the system following

$$
\left\{\begin{array}{l}
\dot{x}_{1}(t)=x_{2}(t), \\
\dot{x}_{2}(t)=R^{2} f\left(x_{1}(t)-v(t), \frac{x_{2}(t)}{R}\right)
\end{array}\right.
$$

satisfies

$$
\lim _{R \rightarrow \infty} \int_{0}^{T}\left|x_{1}(t)-v(t)\right| \mathrm{d} t=0 .
$$

In order to prove Theorem 1.1, the authors of $\mathrm{Su}$ et al. (2005) first prove the following Lemma 1.1 (as Lemma 2 of Su et al. (2005), see also Emaru and Tsuchiya (2003)).

Lemma 1.1 (Su et al. 2005): Theorem 1.1 is true if $v(t)$ is a constant function.

Sketch proof of Theorem 1.1 ( $\mathrm{Su}$ et al. 2005): By Lemma 1.1, the result is true for $v(t)=$ const. For the general bounded integrable $v(t), t \in[0, T]$, consider it

*Corresponding author. Email: gsdxzzl@mail.ustc.edu.cn 
firstly as a function of $L^{1}(0, T)$. For any $\varepsilon>0$, there is a continuous function $\psi \in C[0 T]$ such that

$$
\int_{0}^{T}|v(t)-\psi(t)| \mathrm{d} t<\frac{\varepsilon}{4} .
$$

For this $\psi$, there is a sequence of step functions $\varphi_{n}(t)$, $n=1,2, \ldots$, such that $\varphi_{n}$ converges to $\psi$ uniformly in $[0, T]$. So there exists an integer $N$, such that $\left|\psi(t)-\varphi_{M}(t)\right|<\varepsilon /(4 T)$ as $M>N$ for all $t \in[0 T]$. Therefore,

$$
\begin{aligned}
\int_{0}^{T}\left|v(t)-\varphi_{M}(t)\right| \mathrm{d} t \leq & \int_{0}^{T}|v(t)-\psi(t)| \mathrm{d} t \\
& +\int_{0}^{T}\left|\psi(t)-\varphi_{M}(t)\right| \mathrm{d} t<\frac{\varepsilon}{2} .
\end{aligned}
$$

Suppose that $I_{i}, i=1,2, \ldots, m$ is a partition of $[0, T]$ such that $\varphi_{M}$ takes constant in each $I_{i}$. By Lemma 1.1, there exists an $R_{0}>0$ such that as $R>R_{0}$,

$$
\int_{I_{i}}\left|x_{1}(t)-\varphi_{M}(t)\right| \mathrm{d} t<\frac{\varepsilon}{2 m} \quad \forall i=1,2, \ldots, m .
$$

Consequently,

$$
\int_{0}^{T}\left|x_{1}(t)-\varphi_{M}(t)\right| \mathrm{d} t<\frac{\varepsilon}{2} .
$$

Therefore, as $R>R_{0}$, it has

$$
\begin{aligned}
\int_{0}^{T}\left|x_{1}(t)-v(t)\right| \mathrm{d} t \leq & \int_{0}^{T}\left|x_{1}(t)-\varphi_{M}(t)\right| \mathrm{d} t \\
& +\int_{0}^{T}\left|\varphi_{M}(t)-v(t)\right| \mathrm{d} t<\varepsilon .
\end{aligned}
$$

The proof is complete.

We now indicate where the mistake takes place in the proof of Theorem 1.1. Firstly, the Lemma 1.1 is only true for any given initial value of (1.2). Secondly, in the first interval $I_{1}$, we can find an $R_{1}$ such that (1.4) is true for all $R>R_{1}$. But in the second interval $I_{2}$, the initial value of Equation (1.2) comes from $I_{1}$ that depends on $R>R_{1}$. So we do not know if we can find a common $R_{2}$ such that (1.4) is true in $I_{2}$ for all these $R$-dependent initial values as $R>R_{2}$. So one could not get simply a common $R_{0}$ as claimed before Equation (1.4). Therefore, the proof of Theorem 1 of $\mathrm{Su}$ et al. (2005) (and Theorem 1 of earlier literature Han (1989) as well) is invalid.

It should be indicated that a first correct proof of Theorem 1.1 for nonlinear system under restrictive condition is given in Wang, Chen, and Yang (2007) where it requires that the system (1.1) has a Lyapunov function $V$ satisfying

- $\dot{V}+c V^{\theta} \leq 0$ in $\mathbb{R}^{2}$ for some $c>0, \theta \in(0,1)$, where $\dot{V}=\nabla_{x} V \cdot f(x), x \in \mathbb{R}^{2}$

- the gradient $\nabla_{x} V$ is bounded in $\mathbb{R}^{2}$.
The first condition above is equivalent to that the zero is a globally finite-time-stable equilibrium: for any given initial value, the solution of system (1.1) would be zero after an initial value dependent finite time. This excludes even the linear case proved in Guo et al. (2002) and is hard to verify for nonlinear systems because the Lyapunov function is not unique.

There are many other research works on differentiation trackers like high-gain observer based differentiator (Dabroom and Khalil 1997), the supertwisting second-order sliding-mode algorithm (Davila, Fridman, and Levant 2005), linear time-derivative tracker (Ibrir 2004), robust exact differentiation (Levant 1998, 2003), to name just a few. However, the tracking differentiator (1.2) has its advantage that (a) it has weak stability; (b) it requires weak condition on the input and (c) it has small integrate value of $\left|x_{1}(t)-v(t)\right|$ in any bounded time interval rather than the small error of $\left|x_{1}(t)-v(t)\right|$ after a finite transient time. For a nice comparison with different differentiation trackers, we refer to Xue, Huang, and Yang (2010) (one problem indicated in Xue et al. (2010) for slidingmode based observer is the chattering problem). Moreover, it is shown by linear case in Guo et al. (2002) that the tracking differentiator (1.2) is noise-tolerant.

In this article, we give a rigorous convergence proof for this tracking differentiator under some additional conditions for smooth systems, which is presented in Section 2. In Section 3, the result of Section 2 is generalised to high-order tracking differentiator. Finally, in Section 4, we give an application of the tracking differentiator to the online frequency estimation of the finite sum of the sinusoidal signals, which generalises the result of Guo et al. (2002). Some numerical simulation results are presented to illustrate the effectiveness of the estimation.

\section{Convergence proof for tracking differentiator}

Our main result is stated as Theorem 2.1.

Theorem 2.1: Let $f: \mathbb{R}^{2} \rightarrow \mathbb{R}$ be a locally Lipschitz continuous function, $f(0,0)=0$. Suppose that the equilibrium point $(0,0)$ of the following system is globally asymptotically stable:

$$
\left\{\begin{array}{l}
\dot{x}_{1}(t)=x_{2}(t), \quad x_{1}(0)=x_{10}, \\
\dot{x}_{2}(t)=f\left(x_{1}(t), x_{2}(t)\right), \quad x_{2}(0)=x_{20},
\end{array}\right.
$$

where $\left(x_{10}, x_{20}\right)$ is any given initial value. That is, the equilibrium point $(0,0)$ is stable in the sense that for any $\varepsilon>0$, there exists a $\delta>0$ such that $\left|x_{1}(t)\right|+\left|x_{2}(t)\right|<\varepsilon$ for all $t>0$ as long as $\left|x_{10}\right|+\left|x_{20}\right|<\delta$. Moreover, $\left(x_{1}(t) x_{2}(t)\right) \rightarrow 0$ as $t \rightarrow \infty$. 
If the signal $v$ is differentiable and $A=\sup _{t \in[0, \infty)}|\dot{v}(t)|<\infty$, then the solution of the following tracking differentiator:

$$
\left\{\begin{array}{l}
\dot{z}_{1 R}(t)=z_{2 R}(t), \quad z_{1 R}(0)=z_{10}, \\
\dot{z}_{2 R}(t)=R^{2} f\left(z_{1 R}(t)-v(t), \frac{z_{2 R}(t)}{R}\right), \quad z_{2 R}(0)=z_{20}
\end{array}\right.
$$

is convergent in the sense that: for every $a>0, z_{1 R}$ is uniformly convergent to $v$ on $[a, \infty)$ as $R \rightarrow \infty$, where $\left(z_{10}, z_{20}\right)$ is any given initial value.

Proof: The proof will be split into several steps.

Step 1: Transform the system (2.2) into the system (2.1) with a perturbation.

Suppose that $\left(z_{1 R}, z_{2 R}\right)$ is the solution of system (2.2). Let $t=\frac{s}{R}$. Then

$$
\left\{\begin{array}{l}
\frac{\mathrm{d}}{\mathrm{d} s} z_{1 R}\left(\frac{s}{R}\right)=\frac{1}{R} z_{1 R}^{\prime}\left(\frac{s}{R}\right)=\frac{1}{R} z_{2 R}\left(\frac{s}{R}\right), \\
\frac{\mathrm{d}}{\mathrm{d} s} z_{2 R}\left(\frac{s}{R}\right)=\frac{1}{R} z_{2 R}^{\prime}\left(\frac{s}{R}\right)=R f\left(z_{1 R}\left(\frac{s}{R}\right)-v\left(\frac{s}{R}\right), \frac{z_{2 R}\left(\frac{s}{R}\right)}{R}\right) .
\end{array}\right.
$$

Let

$$
\left\{\begin{array}{l}
y_{1 R}(s)=z_{1 R}\left(\frac{s}{R}\right)-v\left(\frac{s}{R}\right) \\
y_{2 R}(s)=\frac{1}{R} z_{2 R}\left(\frac{s}{R}\right)
\end{array}\right.
$$

Then

$$
\left\{\begin{array}{l}
\dot{y}_{1 R}(s)=y_{2 R}(s)-\frac{\dot{v}\left(\frac{s}{R}\right)}{R}, \quad y_{1 R}(0)=z_{1 R}(0)-v(0), \\
\dot{y}_{2 R}(s)=f\left(y_{1 R}(s), y_{2 R}(s)\right), \quad y_{2 R}(0)=\frac{z_{2 R}(0)}{R} .
\end{array}\right.
$$

Therefore, $Y_{R}=\left(y_{1 R}, y_{2 R}\right)^{\top}$ is a solution to the system

$$
\begin{aligned}
& \dot{Y}_{R}(t)=F\left(Y_{R}(t)\right)+G_{R}(t), \\
& Y_{R}(0)=Y_{R 0}=\left(z_{1 R}(0)-v(0), \frac{z_{2 R}(0)}{R}\right)^{\top},
\end{aligned}
$$

where

$$
\begin{aligned}
F\left(Y_{R}(t)\right) & =\left(y_{2 R}(t), f\left(y_{1 R}(t), y_{2 R}(t)\right)\right)^{\top}, \\
G_{R}(t) & =\left(-\frac{\dot{v}\left(\frac{t}{R}\right)}{R}, 0\right)^{\top} .
\end{aligned}
$$

If $X=\left(x_{1}, x_{2}\right)^{\top}$ is a solution to the system (2.1), then (2.1) can be written as

$$
\dot{X}(t)=F(X(t)) \text {. }
$$

It is seen that the system (2.5) is a perturbed system of (2.6).

Step 2: The existence of Lyapunov function.
Since $f$ is locally Lipschitz continuous and system (2.1) is globally asymptotically stable, by a result of inverse-like Lyapunov theorem in ordinary differential equations (see Theorem 4.17 of Khalil (2002, p. 167)) that there is a smooth, positive definite function $V: \mathbb{R}^{2} \rightarrow \mathbb{R}$ and a continuous, positive definite function $W: \mathbb{R}^{2} \rightarrow \mathbb{R}$ such that

- $V\left(x_{1}, x_{2}\right) \rightarrow \infty$ as $\left|\left(x_{1}, x_{2}\right)\right| \rightarrow \infty$;

- $\frac{\mathrm{d} V}{\mathrm{~d} t}=x_{2} \frac{\partial V}{\partial x_{1}}+f\left(x_{1}, x_{2}\right) \frac{\partial V}{\partial x_{2}} \leq-W\left(x_{1}, x_{2}\right)$

along the trajectory of (2.1);

- $\left\{\left(x_{1}, x_{2}\right) \in \mathbb{R}^{2} \mid V\left(x_{1}, x_{2}\right) \leq d\right\}$

is a bounded closed set of $\mathbb{R}^{2}$ for any given $d>0$.

By the existence of above continuous positive definite functions, it follows from Lemma 4.3 of Khalil (2002, p. 145) that there exist wedge functions $K_{i}:[0, \infty) \rightarrow[0, \infty), i=1,2,3,4$ such that

$$
\begin{aligned}
K_{1}\left(\left|\left(x_{1}, x_{2}\right)\right|\right) & \leq V\left(x_{1}, x_{2}\right) \leq K_{2}\left(\left|\left(x_{1}, x_{2}\right)\right|\right), \\
\lim _{r \rightarrow \infty} K_{i}(r) & =\infty, \quad i=1,2, \\
K_{3}\left(\left|\left(x_{1}, x_{2}\right)\right|\right) & \leq W\left(x_{1}, x_{2}\right) \leq K_{4}\left(\left|\left(x_{1}, x_{2}\right)\right|\right) .
\end{aligned}
$$

Denote by $Y_{R}\left(t ; 0, Y_{R 0}\right)$ the solution of (2.5).

Step 3: For each $Y_{R 0} \in \mathbb{R}^{2}$, there exists an $R_{1}>1$ such that when $R>R_{1}$,

$$
\begin{aligned}
& \left\{Y_{R}\left(t ; 0, Y_{R 0}\right) \mid t \in[0, \infty)\right\} \subset\left\{Y=\left(y_{1}, y_{2}\right) \mid V(Y) \leq c\right\} \\
& \quad c=\max \left\{K_{2}\left(\left|Y_{10}\right|\right), 1\right\}>0 .
\end{aligned}
$$

We assume this claim is false and obtain a contradiction. Firstly, since $\frac{\partial V}{\partial y_{1}}$ is continuous and the set $\{Y \mid c \leq V(Y) \leq c+1\}$ is bounded, we have

$$
M=\sup _{Y \in\{Y \mid c \leq V(Y) \leq c+1\}}\left|\frac{\partial V(Y)}{\partial y_{1}}\right|<\infty .
$$

Secondly,

$$
\begin{gathered}
W(Y) \geq K_{3}(|Y|) \geq K_{3} K_{2}^{-1}(V(Y)) \geq K_{3} K_{2}^{-1}(c)>0 \\
\forall Y \in\{Y \mid c \leq V(Y) \leq c+1\} .
\end{gathered}
$$

Since the claim (2.7) is false and $V\left(Y_{R 0}\right) \leq K_{2}\left(\left|Y_{R 0}\right|\right) \leq K_{2}\left(\left|Y_{10}\right|\right) \leq c$, for $R_{1}$ given by

$$
R_{1}=\max \left\{1, \frac{A M}{K_{3} K_{2}^{-1}(c)}\right\}
$$

there exists an $R>R_{1}$ and $0 \leq t_{1}^{R}<t_{2}^{R}<\infty$ such that

$$
\begin{aligned}
& Y_{R}\left(t_{1}^{R} ; 0, Y_{R 0}\right) \in\{Y \mid V(Y)=c\}, \\
& Y_{R}\left(t_{2}^{R} ; 0, Y_{R 0}\right) \in\{Y \mid V(Y)>c\},
\end{aligned}
$$


and

$$
\left\{Y_{R}\left(t ; 0, Y_{R 0}\right) \mid t \in\left[t_{1}^{R}, t_{2}^{R}\right]\right\} \subset\{Y \mid c \leq V(Y) \leq c+1\} .
$$

Combining (2.8) and (2.11) yields

$$
\inf _{t \in\left[t_{1}^{R}, t_{2}^{R}\right]} W\left(Y_{R}\left(t ; 0, Y_{R 0}\right)\right) \geq K_{3} K_{2}^{-1}(c) .
$$

Therefore, for $t \in\left[t_{1}^{R}, t_{2}^{R}\right]$,

$$
\begin{aligned}
\frac{\mathrm{d} V\left(Y_{R}\left(t ; 0, Y_{R 0}\right)\right)}{\mathrm{d} t} & =\left.\frac{\mathrm{d} V}{\mathrm{~d} t}\right|_{\text {along the system }(2.5)} \\
& \leq-W\left(Y_{R}\left(t ; 0, Y_{R 0}\right)\right)+\frac{A M}{R} \\
& \leq-K_{3} K_{2}^{-1}(c)+A M \frac{K_{3} K_{2}^{-1}(c)}{A M} \\
& =0,
\end{aligned}
$$

which shows that $V\left(Y_{R}\left(t ; 0, Y_{R 0}\right)\right)$ is non-increasing in $\left[t_{1}^{R}, t_{2}^{R}\right]$, and hence

$$
V\left(Y_{R}\left(t_{2}^{R} ; 0, Y_{R 0}\right)\right) \leq V\left(Y_{R}\left(t_{1}^{R} ; 0, Y_{R 0}\right)\right)=c .
$$

This contradicts (2.10), and hence (2.7) is valid.

Step 4: There is an $R_{2} \geq R_{1}$, such that for each $R>R_{2}$, there exists a $T_{R} \in\left[0, \frac{2 c}{K_{3} K_{2}^{-1}(\delta)}\right]$ such that $\left|Y_{R}\left(T_{R} ; 0, Y_{R 0}\right)\right|<\delta$.

Actually, for any given $\varepsilon>0$, since $V$ is continuous, there exists a $\delta \in(0, \varepsilon)$ such that

$$
0 \leq V(Y) \leq K_{1}(\varepsilon) \quad \forall|Y| \leq \delta .
$$

Now, for each $Y \in\{Y|| V(Y) \mid \geq \delta\}$,

$$
W(Y) \geq K_{3}(|Y|) \geq K_{3} K_{2}^{-1}(V(Y)) \geq K_{3} K_{2}^{-1}(\delta)>0 .
$$

By Step 3, for every $R>R_{1}, \quad\left\{Y_{R}\left(t ; 0, Y_{R 0}\right) \mid\right.$ $t \in[0, \infty)\} \subset\{Y \mid V(Y) \leq c\}$, and hence

$$
H=\sup _{t \in[0, \infty)}\left|\frac{\partial V}{\partial y_{1}}\left(Y_{R}\left(t ; 0, Y_{R 0}\right)\right)\right| \leq \sup _{Y \in\{Y \mid V(Y) \leq c\}}\left|\frac{\partial V}{\partial y_{1}}(Y)\right|<\infty .
$$

Suppose that the claim is false. Then for

$$
R_{2}=\max \left\{R_{1}, \frac{2 H A}{K_{3} K_{2}^{-1}(\delta)}\right\},
$$

there exists an $R>R_{2}$ such that $\left|Y_{R}\left(t ; 0, Y_{R 0}\right)\right| \geq \delta$ for any $t \in\left[0, \frac{2 c}{K_{3} K_{2}^{-1}(\delta)}\right]$. This together with (2.14) concludes that for any $R>R_{2}$ and all $t \in\left[0, \frac{2 c}{K_{3} K_{2}^{-1}(\delta)}\right]$,

$$
\begin{aligned}
\frac{\mathrm{d} V\left(Y_{R}\left(t ; 0, Y_{R 0}\right)\right)}{d t}= & \left.\frac{\mathrm{d} V}{\mathrm{~d} t}\right|_{\text {along the system }(2.5)} \\
\leq & -W\left(Y_{R}\left(t ; 0, Y_{R 0}\right)\right) \\
& +\left|\frac{\partial V\left(Y_{R}\left(t ; 0, Y_{R 0}\right)\right)}{\partial y_{1}} \frac{v^{\prime}\left(\frac{t}{R}\right)}{R}\right| \\
\leq & -\frac{K_{3} K_{2}^{-1}(\delta)}{2}<0 .
\end{aligned}
$$

Integrating above inequality over $\left[0, \frac{2 c}{K_{3} K_{2}^{-1}(\delta)}\right]$ to give

$$
\begin{aligned}
& V\left(Y_{R}\left(\frac{2 c}{K_{3} K_{2}^{-1}(\delta)} ; 0, Y_{R 0}\right)\right) \\
& =\int_{0} \frac{2 c}{K_{3} K_{2}^{-1}(\delta)} \frac{\mathrm{d} V\left(Y_{R}\left(t ; 0, Y_{R 0}\right)\right)}{\mathrm{d} t} \mathrm{~d} t+V\left(Y_{R 0}\right) \\
& \leq-\frac{K_{3} K_{2}^{-1}(\delta)}{2} \frac{2 c}{K_{3} K_{2}^{-1}(\delta)}+V\left(Y_{R 0}\right) \\
& \leq 0 .
\end{aligned}
$$

This is a contradiction since for each $t \in\left[0, \frac{2 c}{K_{3} K_{2}^{-1}(\delta)}\right]$, $\left|Y_{R}\left(t ; 0, Y_{R 0}\right)\right| \geq \delta$. The claim follows.

Step 5: For each $R>R_{2}$, if there exists a $t_{0}^{R} \in[0, \infty)$ such that

$$
Y_{R}\left(t_{0}^{R} ; 0, Y_{R 0}\right) \in\{Y|| Y \mid \leq \delta\},
$$

then

$$
\left\{Y_{R}\left(t ; 0, Y_{R 0}\right) \mid t \in\left(t_{0}^{R}, \infty\right)\right\} \subset\{Y \| Y \mid \leq \varepsilon\} .
$$

Suppose (2.16) is not valid. Then there is a $t_{2}^{R}>t_{1}^{R} \geq t_{0}^{R}$ such that

$$
\begin{aligned}
\left|Y_{R}\left(t_{1}^{R} ; 0, Y_{R 0}\right)\right| & =\delta, \\
\left|Y_{R}\left(t_{2}^{R} ; 0, Y_{R 0}\right)\right| & >\varepsilon, \\
\left\{Y_{R}\left(t ; 0, Y_{R 0}\right) \mid t \in\left[t_{1}^{R}, t_{2}^{R}\right]\right\} & \subset\{Y|| Y \mid \geq \delta\} .
\end{aligned}
$$

This together with (2.14) concludes that for $t \in\left[t_{1}^{R}, t_{2}^{R}\right]$,

$$
\begin{aligned}
K_{1}\left(\left|Y_{R}\left(t_{2}^{R} ; 0, Y_{R 0}\right)\right|\right) \leq & V\left(Y\left(t_{2}^{R} ; 0, Y_{R 0}\right)\right) \\
= & \int_{t_{1}^{R}}^{t_{2}^{R}} \frac{\mathrm{d} V\left(Y\left(t ; 0, Y_{R 0}\right)\right)}{\mathrm{d} t} \mathrm{~d} t \\
& +V\left(Y_{R}\left(t_{1}^{R} ; 0, Y_{R 0}\right)\right) \\
\leq & \int_{t_{1}^{R}}^{t_{2}^{R}}-\frac{K_{3} K_{2}^{-1}(\delta)}{2} \mathrm{~d} t \\
& +V\left(Y_{R}\left(t_{1}^{R} ; 0, Y_{R 0}\right)\right) \\
\leq & V\left(Y_{R}\left(t_{1}^{R} ; 0, Y_{R 0}\right)\right) .
\end{aligned}
$$

By (2.13) and $\left|Y_{R}\left(t_{1}^{R} ; 0, Y_{R 0}\right)\right|=\delta$, we have

$$
V\left(Y_{R}\left(t_{1}^{R} ; 0, Y_{R 0}\right)\right) \leq K_{1}(\varepsilon) .
$$

This together with (2.18) gives

$$
K_{1}\left(\left|Y_{R}\left(t_{2}^{R} ; 0, Y_{R 0}\right)\right|\right) \leq K_{1}(\varepsilon) .
$$

Since the wedge function $K_{1}$ is increasing, the above inequality implies $\left|Y_{R}\left(t_{2}^{R} ; 0, Y_{R 0}\right)\right| \leq \varepsilon$, which contradicts the middle inequality of (2.17). The claim (2.16) follows.

Finally, for each $a>0$, by results of Step 4 and Step 5, for $R>\max \left\{R_{2}, \frac{2 c}{a K_{3} K_{2}^{-1}(\delta)}\right\}$ and $t \in[a, \infty)$, 
we have

$$
\left|z_{1 R}(t)-v(t)\right|=\left|y_{1 R}(R t)\right| \leq\left|Y_{R}(R t)\right| \leq \varepsilon .
$$

Hence $z_{1 R}$ converges uniformly to $v$ in $[a, \infty)$ as $R \rightarrow \infty$. The proof is complete.

\section{High-order tracking differentiator}

In order to get the approximations of the high-order derivatives of a signal, we need the high-order tracking differentiator. The following Theorem 3.1 is about the convergence of high-order tracking differentiator.

Theorem 3.1: Let $f: \mathbb{R}^{n} \rightarrow \mathbb{R}$ be a locally Lipschitz continuous function. Assume that the equilibrium point $(0,0, \ldots, 0)$ of the following system is globally asymptotically stable:

$$
\left\{\begin{array}{l}
\dot{x}_{1}(t)=x_{2}(t), x_{1}(0)=x_{10}, \\
\dot{x}_{2}(t)=x_{3}(t), x_{2}(0)=x_{20}, \\
\cdots \cdots \cdots \cdots \\
\dot{x}_{n-1}(t)=x_{n}(t), x_{n-1}(0)=x_{(n-1) 0}, \\
\dot{x}_{n}(t)=f\left(x_{1}(t), x_{2}(t), \ldots, x_{n}(t)\right), x_{n}(0)=x_{n 0},
\end{array}\right.
$$

where $\left(x_{10}, x_{20}, \ldots, x_{n 0}\right)$ is any given initial value. If the signal $v$ is differentiable and $A=\sup _{t \in[0, \infty)}|\dot{v}(t)|<\infty$, then the solution of the following tracking differentiator:

$$
\left\{\begin{array}{l}
\dot{z}_{1 R}(t)=z_{2 R}(t), z_{1 R}(0)=z_{10}, \\
\dot{z}_{2 R}(t)=z_{3 R}(t), z_{2 R}(0)=z_{20}, \\
\cdots \cdots \\
\dot{z}_{(n-1) R}(t)=z_{n R}(t), \quad z_{(n-1) R}(0)=z_{(n-1) 0}, \\
\dot{z}_{n R}(t)=R^{n} f\left(z_{1 R}(t)-v(t), \frac{z_{2 R}(t)}{R}, \ldots, \frac{z_{n R}(t)}{R^{n-1}}\right), \\
z_{n R}(0)=z_{n 0}
\end{array}\right.
$$

is convergent in the sense that: for every $a>0, z_{1 R}$ is uniformly convergent to $v$ on $[a, \infty)$ as $R \rightarrow \infty$, where $\left(z_{10}, z_{20}, \ldots, z_{n 0}\right)$ is any given initial value.

Proof: Suppose that $\left(z_{1 R}, z_{2 R}, \ldots, z_{n R}\right)$ is the solution of system (3.2). Let $t=\frac{s}{R}$. Then

$$
\left\{\begin{array}{l}
\frac{\mathrm{d}}{\mathrm{d} s} z_{1 R}\left(\frac{s}{R}\right)=\frac{1}{R} z_{1 R}^{\prime}\left(\frac{s}{R}\right)=\frac{1}{R} z_{2 R}\left(\frac{s}{R}\right), \\
\frac{\mathrm{d}}{\mathrm{d} s} z_{2 R}\left(\frac{s}{R}\right)=\frac{1}{R} z_{2 R}^{\prime}\left(\frac{s}{R}\right)=\frac{1}{R} z_{3 R}\left(\frac{s}{R}\right), \\
\cdots \ldots \ldots \ldots \ldots \ldots \ldots \ldots \ldots \ldots \ldots \ldots \ldots \ldots \ldots \ldots \ldots \\
\frac{\mathrm{d}}{\mathrm{d} s} z_{(n-1) R}\left(\frac{s}{R}\right)=\frac{1}{R} z_{(n-1) R}^{\prime}\left(\frac{s}{R}\right)=\frac{1}{R} z_{n R}\left(\frac{s}{R}\right), \\
\frac{\mathrm{d}}{\mathrm{d} s} z_{n R}\left(\frac{s}{R}\right)=\frac{1}{R} z_{n R}^{\prime}\left(\frac{s}{R}\right)=R^{n-1} f\left(z_{1 R}\left(\frac{s}{R}\right)-v\left(\frac{s}{R}\right),\right. \\
\left.z_{2 R}\left(\frac{s}{R}\right), \ldots, z_{n R}\left(\frac{s}{R}\right)\right) .
\end{array}\right.
$$

Let

$$
\left\{\begin{array}{l}
y_{1 R}(s)=z_{1 R}\left(\frac{s}{R}\right)-v\left(\frac{s}{R}\right), \\
y_{2 R}(s)=\frac{1}{R} z_{2 R}\left(\frac{s}{R}\right), \\
y_{3 R}(s)=\frac{1}{R^{2}} z_{3 R}\left(\frac{s}{R}\right), \\
\ldots \ldots \ldots \cdots \cdots \cdots \\
y_{n R}(s)=\frac{1}{R^{n-1}} z_{n R}\left(\frac{s}{R}\right) .
\end{array}\right.
$$

Then

$$
\left\{\begin{array}{l}
\dot{y}_{1 R}(s)=y_{2 R}(s)-\frac{\dot{v}\left(\frac{s}{R}\right)}{R}, \\
\dot{y}_{2 R}(s)=y_{3 R}(s), \\
\cdots \cdots \cdots \cdots \cdots \cdots \cdots \cdots \cdots \cdots \cdots \\
\dot{y}_{(n-1) R}(s)=y_{n R}(s) \\
\dot{y}_{n R}(s)=f\left(y_{1 R}(s), y_{2 R}(s), \ldots, y_{n R}(s)\right) .
\end{array}\right.
$$

Therefore, $\left(y_{1 R}, y_{2 R}, \ldots, y_{n R}\right)$ is a solution to the disturbed system of (3.1). Again we can write (3.4) as

$$
\dot{Y}_{R}(t)=F\left(Y_{R}(t)\right)+G_{R}(t),
$$

where $Y_{R}=\left(y_{1 R}, y_{2 R}, \ldots, y_{n R}\right)^{\top}$, and

$$
\begin{aligned}
F\left(Y_{R}(t)\right) & =\left(y_{2 R}(t), \ldots, y_{n R}(t), f\left(y_{1 R}(t), y_{2 R}(t), \ldots, y_{n R}(t)\right)\right)^{\top}, \\
G_{R}(t) & =\left(-\frac{\dot{v}\left(\frac{t}{R}\right)}{R}, 0, \ldots, 0\right)^{\top} .
\end{aligned}
$$

It is seen that except the dimension, there is no difference between (2.5) and (3.5). Therefore, the proof for the theorem can now be completed along the same line of the proof of Theorem 2.1.

Remark 3.1: Because of Theorem 3.1, we can consider $z_{i R}(t)$ as the approximation of the derivative $v^{(i-1)}(t)$ for $i=2,3, \ldots, n$ provided that the latter exist in the classical sense or are considered as the generalised derivatives by considering $v$ as a generalised function.

Theorem 2.1 is first proved in Guo et al. (2002) with linear $f$ where the result is much stronger than that claimed by Theorem 2.1: the $z_{2 R}$ is indeed the approximation of the derivative of $v$ in the classical sense. For the nonlinear $f$, it is still open for the convergence of $z_{2 R}$ to $\dot{v}$ in classical sense. In Theorem 3.2, we generalise the result of Guo et al. (2002) to the higher order tracking differentiator.

Theorem 3.2: Assume that the matrix following

$$
A=\left(\begin{array}{ccccc}
0 & 1 & 0 & \cdots & 0 \\
0 & 0 & 1 & \cdots & 0 \\
\vdots & \vdots & \vdots & \ddots & \vdots \\
0 & 0 & 0 & \cdots & 1 \\
a_{1} & a_{2} & a_{3} & \cdots & a_{n}
\end{array}\right)
$$


is Hurwitz, and $v:[0, \infty) \rightarrow \mathbb{R}$ is the function satisfying $\sup _{t \in[0, T], 1 \leq k \leq n}\left|v^{(k)}(t)\right|=M<\infty$ for constants $T, M>0$. Then the following linear tracking differentiator

$\left\{\begin{array}{l}\dot{z}_{1 R}(t)=z_{2 R}(t), z_{1 R}(0)=z_{10}, \\ \dot{z}_{2 R}(t)=z_{3 R}(t), z_{2 R}(0)=z_{20}, \\ \cdots \cdots \cdots \cdots \cdots \cdots \cdots \cdots \cdots \cdots \cdots \cdots \cdots \\ \dot{z}_{(n-1) R}(t)=z_{n R}(t), z_{(n-1) R}(0)=z_{(n-1) 0}, \\ \dot{z}_{n R}(t)=R^{n}\left(a_{1}\left(z_{1 R}(t)-v(t)\right)+\frac{a_{2} z_{2 R}(t)}{R}+\cdots+\frac{a_{n} z_{n R}(t)}{R^{n-1}}\right), \\ z_{n R}(0)=z_{n 0}\end{array}\right.$

is convergent in the sense that: for any $0<a<T, z_{k R}$ $(k=1,2, \ldots, n)$ converges uniformly to $v^{(k-1)}$ in $[a, T]$ as $R \rightarrow \infty$, where $\left(z_{10}, z_{20}, \ldots, z_{n 0}\right)$ is any given initial value.

Proof: In the linear case, (3.5) becomes

$$
\dot{Y}_{R}(t)=A Y_{R}(t)+\left[\frac{\dot{v}\left(\frac{t}{R}\right)}{R}, 0, \ldots, 0\right]^{\top} .
$$

Solve the linear differential equation (3.8) to get

$$
Y_{R}(t)=e^{A t} Y_{R}(0)+\int_{0}^{t} e^{A(t-s)}\left[\frac{\dot{v}\left(\frac{s}{R}\right)}{R}, 0, \ldots, 0\right]^{\top} \mathrm{d} s .
$$

It then follows that

$$
y_{1 R}(t)=\left[e^{A t}\right]_{1} Y_{R}(0)+\int_{0}^{t}\left[e^{A(t-s)}\right]_{11} \frac{\dot{v}\left(\frac{S}{R}\right)}{R} \mathrm{~d} s,
$$

where $\left[e^{A t}\right]_{1}$ denotes the first row of the matrix $e^{A t}$, and $\left[e^{A(t-s)}\right]_{11}$ the first entry of $e^{A(t-s)}$.

By (3.3) and (3.10), we have

$$
z_{1 R}(t)=\left[e^{R A t}\right]_{1} Y_{R}(0)+\int_{0}^{R t}\left[e^{A(R t-s)}\right]_{11} \frac{\dot{v}\left(\frac{S}{R}\right)}{R} \mathrm{~d} s+v(t) .
$$

Differentiate $z_{1 R}$ with respect to $t$ to give

$$
\begin{aligned}
z_{2 R}(t)= & \dot{z}_{1 R}(t) \\
= & {\left[R A e^{R A t}\right]_{1} Y_{R}(0)+\dot{v}(t) } \\
& +\int_{0}^{R t} \frac{\mathrm{d}}{\mathrm{d} t}\left(\left[e^{A(R t-s)}\right]_{11}\right) \frac{\dot{v}\left(\frac{s}{R}\right)}{R} \mathrm{~d} s+\dot{v}(t) \\
= & {\left[R A e^{R A t}\right]_{1} Y_{R}(0)+\dot{v}(t) } \\
& -\int_{0}^{R t} \frac{\mathrm{d}}{\mathrm{d} s}\left(\left[e^{A(R t-s)}\right]_{11}\right) \dot{v}\left(\frac{s}{R}\right) \mathrm{d} s+\dot{v}(t) \\
= & {\left[R A e^{R A t}\right]_{1} Y_{R}(0)+\dot{v}(t)-\left.\left[e^{A(R t-s)}\right]_{11} \dot{v}\left(\frac{s}{R}\right)\right|_{0} ^{R t} } \\
& +\int_{0}^{R t}\left[e^{A(R t-s)}\right]_{11} \frac{\ddot{v}\left(\frac{s}{R}\right)}{R} \mathrm{~d} s+\dot{v}(t) \\
= & {\left[R A e^{R A t}\right]_{1} Y_{R}(0)+\left[e^{R A t}\right]_{11} \dot{v}(0) } \\
& +\int_{0}^{R t}\left[e^{A(R t-s)}\right]_{11} \frac{\ddot{v}\left(\frac{s}{R}\right)}{R} \mathrm{~d} s+\dot{v}(t) .
\end{aligned}
$$

Generally, we have, by induction that

$$
\begin{aligned}
z_{k R}(t)= & {\left[(R A)^{k-1} e^{R A t}\right]_{1} Y_{R}(0)+\left[(R A)^{k-2} e^{A R t}\right]_{11} \dot{v}(0) } \\
& +\cdots+\left[e^{A R t}\right]_{11} v^{(k-1)}(0) \\
& +\int_{0}^{R t}\left[e^{A(R t-s)}\right]_{11} \frac{v^{(k)}\left(\frac{s}{R}\right)}{R} \mathrm{~d} s+v^{(k-1)}(t), \quad 2 \leq k \leq n .
\end{aligned}
$$

Since $A$ is Hurwitz, we may assume without loss of generality that there exist constants $L, \omega>0$ such that all entries of $e^{A t}=\left\{e_{i j}(t)\right\}_{i, j=1}^{n}$ satisfy

$$
\left|e_{i j}(t)\right| \leq L e^{-\omega t} \quad \forall t \geq 0, i, j=1,2, \ldots, n .
$$

Since $\left|v^{(k)}(t)\right| \leq M \quad \forall t \in[0 T]$, we have, for every $t \in[0, T]$, that

$$
\begin{aligned}
\left|\int_{0}^{R t}\left[e^{A(R t-s)}\right]_{11} \frac{v^{(k)}\left(\frac{s}{R}\right)}{R} \mathrm{~d} s\right| & =\left|\int_{0}^{R t} e_{11}(R t-s) \frac{v^{(k)}\left(\frac{s}{R}\right)}{R} \mathrm{~d} s\right| \\
& \leq \frac{M L}{R} \int_{0}^{R t} e^{-\omega(R t-s)} \mathrm{d} s \leq \frac{M L}{\omega R} .
\end{aligned}
$$

This together with (3.13) and (3.14) gives

$\lim _{R \rightarrow \infty} z_{k R}(t)=v^{(k-1)}(t)$ uniformly in $[a, T]$ for any

$$
0<a<T, \quad 2 \leq k \leq n .
$$

The proof is complete.

Remark 3.2: If $\sup _{t \in[0, \infty], 1 \leq k \leq n}\left|v^{(k)}(t)\right|=M<\infty$, then the conclusion of Theorem 3.2 can be replaced by the more strong form: $z_{k R}(k=1,2, \ldots, n)$ converges uniformly to $v^{(k-1)}$ in $[a, \infty)$, as $R \rightarrow \infty$.

\section{Application to frequency estimation of sinusoidal signals}

We consider the finite sum of sinusoidal signals $v(t)=\sum_{i=1}^{n} A_{i} \sin \left(\omega_{i} t+\phi_{i}\right)$, where the $\omega_{i}>0$ are different frequencies. The aim of this section is to estimate all frequencies $\omega_{i}$ by using tracking-differentiator. The even order derivatives of $v$ in $t$ up to $2 n-2$ are found to be

$$
\begin{gathered}
\ddot{v}(t)=\sum_{i=1}^{n} \theta_{i} A_{i} \sin \left(\omega_{i} t+\phi_{i}\right), \\
v^{(4)}(t)=\sum_{i=1}^{n} \theta_{i}^{2} A_{i} \sin \left(\omega_{i} t+\phi_{i}\right), \\
\ldots \ldots \ldots \ldots \ldots \ldots \ldots \ldots \ldots \ldots \ldots \\
v^{(2 n-2)}(t)=\sum_{i=1}^{n} \theta_{i}^{n-1} A_{i} \sin \left(\omega_{i} t+\phi_{i}\right),
\end{gathered}
$$


where $\theta_{i}=-\omega_{i}^{2}$. That is

$$
\left(\begin{array}{c}
v \\
\ddot{v} \\
\vdots \\
v^{(2 n-2)}
\end{array}\right)=\Lambda\left(\begin{array}{c}
A_{1} \sin \left(\omega_{1} t+\phi_{1}\right) \\
A_{2} \sin \left(\omega_{2} t+\phi_{2}\right) \\
\vdots \\
A_{n} \sin \left(\omega_{n} t+\phi_{n}\right)
\end{array}\right) \text {, }
$$

where

$$
\Lambda=\left(\begin{array}{cccc}
1 & 1 & \cdots & 1 \\
\theta_{1} & \theta_{2} & \cdots & \theta_{n} \\
\cdots & \cdots & \ddots & \cdots \\
\theta_{1}^{n-1} & \theta_{2}^{n-1} & \cdots & \theta_{n}^{n-1}
\end{array}\right)
$$

Since $\Lambda$ is invertible, we have

$$
\left(\begin{array}{c}
A_{1} \sin \left(\omega_{1} t+\phi_{1}\right) \\
A_{2} \sin \left(\omega_{2} t+\phi_{2}\right) \\
\vdots \\
A_{n} \sin \left(\omega_{n} t+\phi_{n}\right)
\end{array}\right)=\Lambda^{-1}\left(\begin{array}{c}
v \\
\ddot{v} \\
\vdots \\
v^{(2 n-2)}
\end{array}\right)
$$

Denote $\Lambda^{-1}$ by

$$
\Lambda^{-1}=\left(\begin{array}{cccc}
\lambda_{11} & \lambda_{12} & \cdots & \lambda_{1 n} \\
\lambda_{21} & \lambda_{22} & \cdots & \lambda_{2 n} \\
\cdots & \cdots & \ddots & \cdots \\
\lambda_{n 1} & \lambda_{n 2} & \cdots & \lambda_{n n}
\end{array}\right)
$$

Since for any $b \geq 0$

$$
\omega_{k}^{2}=\lim _{t \rightarrow \infty} \frac{\int_{b}^{t}\left(\left(A_{k} \sin \left(\omega_{k} t+\phi_{k}\right)\right)^{\prime}\right)^{2} \mathrm{~d} t}{\int_{b}^{t}\left(A_{k} \sin \left(\omega_{k} t+\phi_{k}\right)\right)^{2} \mathrm{~d} t}, \quad k=1,2, \ldots, n,
$$

it follows from (4.3) that

$$
\begin{gathered}
-\theta_{k}=\lim _{t \rightarrow \infty} \frac{\sum_{i, j=1}^{n} \lambda_{k i} \lambda_{k j} \int_{b}^{t} v^{(2 i-1)}(s) v^{(2 j-1)}(s) \mathrm{d} s}{\sum_{i, j=1}^{n} \lambda_{k i} \lambda_{k j} \int_{b}^{t} v^{(2 i-2)}(s) v^{(2 j-2)}(s) \mathrm{d} s}, \\
k=1,2, \ldots, n .
\end{gathered}
$$

Let $T$ be a sufficiently large number, and let

$$
\left\{\begin{array}{l}
a_{i j}=\int_{b}^{T} v^{(2 i-1)}(s) v^{(2 j-1)}(s) \mathrm{d} s, \\
b_{i j}=\int_{b}^{T} v^{(2 i-2)}(s) v^{(2 j-2)}(s) \mathrm{d} s, \quad i, j=1,2, \ldots, n .
\end{array}\right.
$$

By solving the following high-order equations of $n$ unknown elements $\theta_{i}, i=1,2, \ldots, n$, we can get the approximate values of $\theta_{i}$ :

$$
\left\{\begin{array}{l}
\sum_{i, j=1}^{n} \lambda_{1 i} \lambda_{1 j} b_{i j} \theta_{1}+\sum_{i, j=1}^{n} \lambda_{1 i} \lambda_{1 j} a_{1 j}=0, \\
\sum_{i, j=1}^{n} \lambda_{2 i} \lambda_{2 j} b_{i j} \theta_{2}+\sum_{i, j=1}^{n} \lambda_{2 i} \lambda_{2 j} a_{2 j}=0, \ldots \ldots \ldots \ldots \ldots \ldots \ldots \ldots \ldots \ldots \ldots \ldots \ldots \ldots \ldots \ldots \\
\cdots \\
\sum_{i, j=1}^{n} \lambda_{n i} \lambda_{n j} b_{n j} \theta_{1}+\sum_{i, j=1}^{n} \lambda_{n i} \lambda_{n j} a_{n j}=0,
\end{array}\right.
$$

where $\theta_{i}=-\omega_{i}^{2}, \lambda_{i j}$ are rational functions of $\theta_{i}$, and the values of $a_{i j}, b_{i j}$ in (4.5) can be approximated by the high-order tracking differentiator (3.2).

Example 4.1: Let us investigate the two different frequencies case.

$$
v(t)=A_{1} \sin \left(\omega_{1} t+\phi_{1}\right)+A_{2} \sin \left(\omega_{2} t+\phi_{2}\right) .
$$

This is the case of $n=2$. Equation (4.6) now becomes

$$
\left\{\begin{array}{c}
\left(a_{11}+2 b_{12}\right) \theta_{1} \theta_{2}-b_{22} \theta_{1}-b_{22} \theta_{2}-a_{22}=0, \\
b_{11} \theta_{1} \theta_{2}+a_{11} \theta_{2}+a_{11} \theta_{1}-2 a_{12}-b_{22}=0,
\end{array}\right.
$$

and (4.5) becomes, in this case

$$
\left\{\begin{array}{l}
a_{11}=\int_{b}^{T} z_{2 R}^{2}(t) \mathrm{d} t, \quad a_{12}=a_{21}=\int_{b}^{T} z_{2 R}(t) z_{4 R}(t) \mathrm{d} t \\
a_{22}=\int_{b}^{T} z_{4 R}^{2}(t) \mathrm{d} t, \\
b_{11}=\int_{b}^{T} z_{1 R}^{2}(t) \mathrm{d} t, \quad b_{12}=b_{21}=\int_{b}^{T} z_{1 R}(t) z_{3 R}(t) \mathrm{d} t \\
b_{22}=\int_{b}^{T} z_{3 R}^{2}(t) \mathrm{d} t,
\end{array}\right.
$$

where instead of $v^{(i-1)}$, we used directly $z_{i R}$ to be the approximation of $v^{(i-1)}, i=1,2,3,4$, by the linear tracking differentiator (3.7):

$$
\left\{\begin{aligned}
& \dot{z}_{1 R}(t)=z_{2 R}(t), \quad z_{1 R}(0)=z_{10} \\
& \dot{z}_{2 R}(t)=z_{3 R}(t), \quad z_{2 R}(0)=z_{20} \\
& \dot{z}_{3 R}(t)= z_{4 R}(t), \quad z_{3 R}(0)=z_{30} \\
& \dot{z}_{4 R}(t)=-24 R^{4}\left(z_{1 R}(t)-v(t)\right)-50 R^{3} z_{2 R}(t) \\
&-35 R^{2} z_{3 R}(t)-10 R z_{4 R}(t), \quad z_{4 R}(0)=z_{40}
\end{aligned}\right.
$$

Now the matrix $A$ becomes

$$
\left(\begin{array}{cccc}
0 & 1 & 0 & 0 \\
0 & 0 & 1 & 0 \\
0 & 0 & 0 & 1 \\
-24 & -50 & -35 & -10
\end{array}\right)
$$

which has eigenvalues $-1,-2,-3,-4$. So it is Hurwitz. Hence the tracking differentiator (4.10) is well-defined. Note that in (4.8), $\theta_{1}$ and $\theta_{2}$ are symmetrical. If we cancel $\theta_{1}$ from (4.8), we get a quadratic equation of $\theta_{2}$. So if there are two real solutions to (4.8), they must be $\left(\theta_{1}, \theta_{2}\right)$.

Let $A_{1}=1, A_{2}=2, \omega_{1}=1, \omega_{2}=2, \phi_{1}=\phi_{2}=0$ in (4.7), $b=1, T: 2 \rightarrow 35$ with step equal to 0.1 in (4.9) and $z_{10}=z_{20}=z_{30}=z_{40}=0, \quad R=20$ in (4.10). The numerical results for frequency estimation by 

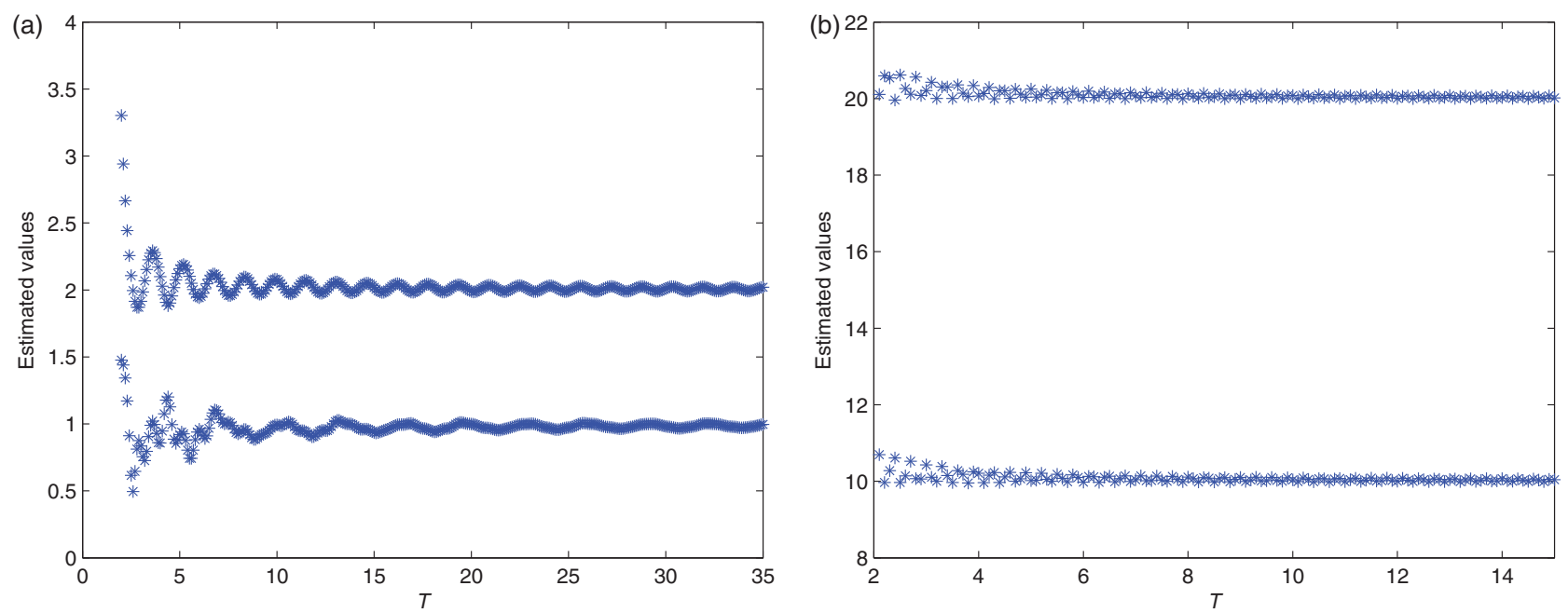

Figure 1. Estimation of one frequency by nonlinear tracking differentiator.

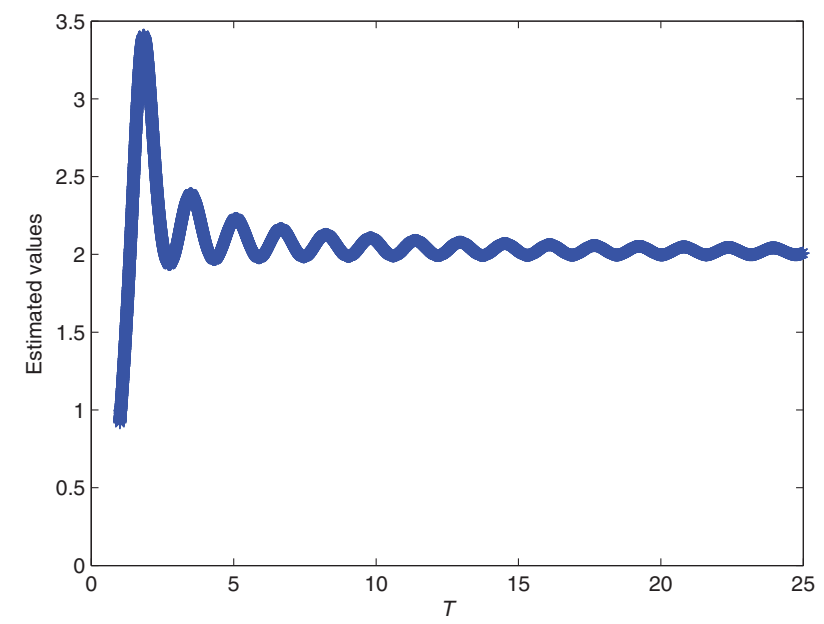

Figure 2. Estimation of two frequencies by linear tracking differentiator.

(4.8)-(4.10) are plotted in Figure 1(a). Figure 1(b) shows the simulation for $A_{1}=A_{2}=1, \omega_{1}=10, \omega_{2}=20$, $\phi_{1}=\phi_{2}=0, \quad z_{10}=z_{20}=z_{30}=z_{40}=0, \quad R=20, \quad b=1$, $T: 2 \rightarrow 15$ with step equal to 0.01 . It is seen that the estimations are quite satisfactory.

Example 4.2: In this example, we use a nonlinear second-order tracking differentiator to estimate the frequency of the signal $v=A \sin (\omega t+\phi)$. This is the case of $n=1$ like that discussed in Guo et al. (2002) by linear tracking differentiator.

In this case

$$
\omega=\lim _{T \rightarrow \infty} \sqrt{\frac{\int_{b}^{T} \dot{v}^{2}(t) \mathrm{d} t}{\int_{b}^{T} v^{2}(t) \mathrm{d} t}} .
$$

The nonlinear tracking differentiator that we use here is

$$
\left\{\begin{aligned}
\dot{z}_{1 R}(t) & =z_{2 R}(t), \quad z_{1 R}(0)=z_{10}, \\
\dot{z}_{2 R}(t) & =-R^{2} \operatorname{sign}\left(z_{1 R}(t)-v(t)\right)\left|z_{1 R}(t)-v(t)\right|^{0.3} \\
& -R z_{2 R}(t), \quad z_{2 R}(0)=z_{20} .
\end{aligned}\right.
$$

In order for the tracking differentiator (4.12) to satisfy all conditions of Theorem 3.1 as (3.2), we only need to prove that the equilibrium point $(0,0)$ of the following systems is globally asymptotically stable:

$$
\left\{\begin{array}{l}
\dot{x}_{1}=x_{2} \\
\dot{x}_{2}=-\operatorname{sign}\left(x_{1}\right)\left|x_{1}\right|^{0.3}-x_{2} .
\end{array}\right.
$$

In fact, let the Lyapunov function be defined by

$$
V\left(x_{1}, x_{2}\right)=\frac{\left|x_{1}\right|^{1.3}}{1.3}+\frac{x_{2}^{2}}{2} .
$$

Then (obviously, this Lyapunov function does not satisfy the condition required in Wang et al. (2007) and is different to the super-twisting observer in Davila et al. (2005) where the function is not Lipschitz continuous)

$$
\left.\frac{\mathrm{d} V}{\mathrm{~d} t}\right|_{\text {along the system (4.13) }}=-x_{2}^{2} \leq 0 .
$$

Note that the set

$$
\left\{\left(x_{1}, x_{2}\right)\left|\frac{\mathrm{d} V}{\mathrm{~d} t}\right|_{\text {along the system (4.13) }}=0\right\}
$$

does not contain any non-zero trajectory of system (4.13). By Corollary 4.1 of Khalil (2002, p. 128), 
the equilibrium point $(0,0)$ of the system (4.13) is globally asymptotically stable. Hence the tracking differentiator (4.12) satisfies all conditions of Theorem 3.1.

In Figure 2, we plot the numerical results for $A=1$, $z_{10}=z_{20}=0, \omega=2, b=10, R=100, T: 10 \rightarrow 25$ with step $=0.0001$. It is obviously convergent.

A different approach by nonlinear observer for frequency estimation of finite sum of the sinusoidal signals is also discussed in Xia (2002). However, due to different approaches, it is hard to compare the effectiveness of these two approaches. But our approach is at least as simple as nonlinear observer. The first far more simple global convergent frequency estimator for single sinusoidal is presented in $\mathrm{Hsu}$, Ortega, and Damm (1999). The noise tolerance of the tracking differentiator presented in this article has been proved both theoretically and numerically for linear case in Guo et al. (2002).

\section{Concluding remarks}

In this article, we give a rigorous proof of the convergence of the nonlinear tracking differentiators for both two-dimensional and high-dimensional cases under some weak assumptions. Numerous applications are reported for this tracking differentiator proposed two decades ago as a key link towards active disturbance rejection control (Han 2009), and its advantages over the existing ones like anti-chattering and noise tolerance. We also apply this tracking differentiator to the frequency estimation of finite sum of the sinusoidal signals. The simulation shows its effectiveness.

\section{Acknowledgements}

This work was carried out with the support of the National Natural Science Foundation of China, the National Basic Research Program of China (2011CB808002) and the National Research Foundation of South Africa.

\section{References}

Dabroom, A.M., and Khalil, H.K. (1997), 'Discrete-time Implementation of High-gain Observers for Numerical Differentiation', International Journal of Control, 12, 1523-1537.

Davila, J., Fridman, L., and Levant, A. (2005), 'Second-order Sliding-modes Observer for Mechanical Systems', IEEE Trans. Automatic Control, 50, 1785-1789.
Emaru, T., and Tsuchiya, T. (2003), 'Research on Estimating Smoothed Value and Differential Value by using Sliding Mode System', IEEE Transactions on Robotics and Automation, 19, 391-402.

Guo, B.Z., Han, J.Q., and Xi, F.B. (2002), 'Linear Trackingdifferentiator and Application to Online Estimation of the Frequency of a Sinusoidal Signal with Random Noise Perturbation', International Journal of Systems Science, 33, 351-358.

Han, J.Q. (1989), 'Control Theory: Model Approach Or Control Approach', Journal of Systems Science and Mathematical Science, 9, 328-335 (in Chinese).

Han, J.Q. (2009), 'From PID to Active Disturbance Rejection Control', IEEE Transactions on Industrial Electronics, 56, 900-906.

Han, J.Q., and Wang, W. (1994), 'Nonlinear Tracking-differentiator', Journal of Systems Science and Mathematical Science, 14, 177-183 (in Chinese).

Hsu, L., Ortega, R., and Damm, G. (1999), 'A Global Convergent Frequency Estimator', IEEE Transactions on Automatic Control, 44, 698-713.

Ibrir, S. (2004), 'Linear Time-derivative Trackers', Automatica, 40, 397-405.

Levant, A. (1998), 'Robust Exact Differentiation via Sliding Mode Technique', Automatica, 34, 379-384.

Levant, A. (2003), 'Higher Order Sliding Modes, Differentiation and Output Feedback Control', International Journal of Control, 76, 924-941.

Khalil, H.K. (2002), Nonlinear Systems, New Jersey: Prentice Hall.

Silva, G.J., Datta, A., and Bhattacharyya, S.P. (2002), 'New Results on the Synthesis of PID Controllers', IEEE Transactions on Automatic Control, 47, 241-252.

Su, Y., Duan, B.Y., Zheng, C.H., Zhang, Y.F., Chen, G.D., and Mi, J.W. (2005), 'Disturbance-rejection High-precision Motion Control of a Stewart Platform', IEEE Transactions on Control Systems Technology, 12, 364-374.

Su, Y.X., Zheng, C.H., Dong, S., and Duan, B.Y. (2005), 'A Simple Nonlinear Velocity Estimation for High-performance Motion Control', IEEE Transactions on Industrial Electronics, 52, 1661-1169.

Sun, B., and Gao, Z. (2005), 'A DSP-based Active Disturbance Rejection Control Design for a $1-\mathrm{kW}$ H-bridge DCCDC Power Converter', IEEE Transactions on Industrial Electronics, 52, 1271-1277.

Xia, X. (2002), 'Global Frequency Estimation using Adaptive Identifiers', IEEE Transactions on Automatic Control, 47, 1188-1193.

Xue, W., Huang, Y., and Yang, X. (2010), 'What Kinds of System can be used as Tracking-differentiator', in Proceedings of the Chinese Control Conference, pp. 6113-6120.

Wang, X., Chen, Z., and Yang, G. (2007), 'Finite-time-convergent Differentiator Based on Singular Perturbation Technique', IEEE Transactions on Automatic Control, 52, 1731-1737. 